\title{
Experimental Design Modelling and Optimization of Triazine Herbicides Removal With Reduced Graphene Oxide Using Response Surface Method
}

\section{Martina Foschi}

University of L'Aquila Department of Physical and Chemical Sciences: Universita degli Studi dell'Aquila Dipartimento di Scienze Fisiche e Chimiche

\section{Paola Capasso}

University of L'Aquila Department of Physical and Chemical Sciences: Universita degli Studi dell'Aquila Dipartimento di Scienze Fisiche e Chimiche

\section{Maria Anna Maggi}

Hortus Novus srl

\section{Fabrizio Ruggieri}

University of L'Aquila Department of Physical and Chemical Sciences: Universita degli Studi dell'Aquila Dipartimento di Scienze Fisiche e Chimiche

\section{Giulia Fioravanti ( $\boldsymbol{\sigma}$ giulia.fioravanti@univaq.it)}

University of L'Aquila Department of Physical and Chemical Sciences: Universita degli Studi dell'Aquila Dipartimento di Scienze Fisiche e Chimiche https://orcid.org/0000-0002-8653-9925

\section{Research Article}

Keywords: reduced graphene oxide, thermal mild reduction, response surface methodology, triazines removal, Freundlich isotherm.

Posted Date: February 9th, 2021

DOI: https://doi.org/10.21203/rs.3.rs-165892/v1

License: (c) (1) This work is licensed under a Creative Commons Attribution 4.0 International License.

Read Full License 
1 Title: Experimental design modelling and optimization of triazine herbicides removal with reduced graphene oxide using response surface method

Authors: Martina Foschi ${ }^{\mathrm{a}}$, Paola Capasso ${ }^{\mathrm{a}}$, Maria Anna Maggi ${ }^{\mathrm{b}}$, Fabrizio Ruggieri ${ }^{\mathrm{a}}$ and Giulia Fioravanti ${ }^{\mathrm{a}^{*}}$

a) University of L'Aquila, Department of Physical and Chemical Sciences, Via Vetoio, Coppito, L'Aquila (AQ) 67100, 7 Italy.

8 b) Hortus Novus, Via Aldo Moro 28 D, L'Aquila (AQ), 67100, Italy

*Corresponding authors

11 E-mail: giulia.fioravanti@univaq.it

12 Department of Physical and Chemical Sciences

13 University of L'Aquila

14 Via Vetoio - 67100 L'Aquila (AQ) - Italy

15 Tel. +39-0862-434244

16 
Thermally reduced GO films enhance the removal of triazines from water.

44 Optimisation of the adsorbent by DOE coupled with the response surface methodology. Atrazine maximum adsorption capacity of $\mathrm{rGO}$ treated at $110^{\circ} \mathrm{C}$ for $24 \mathrm{~h}$ was $46 \%$. Freundlich and Langmuir models were applied to the triazines adsorption on rGO films. 


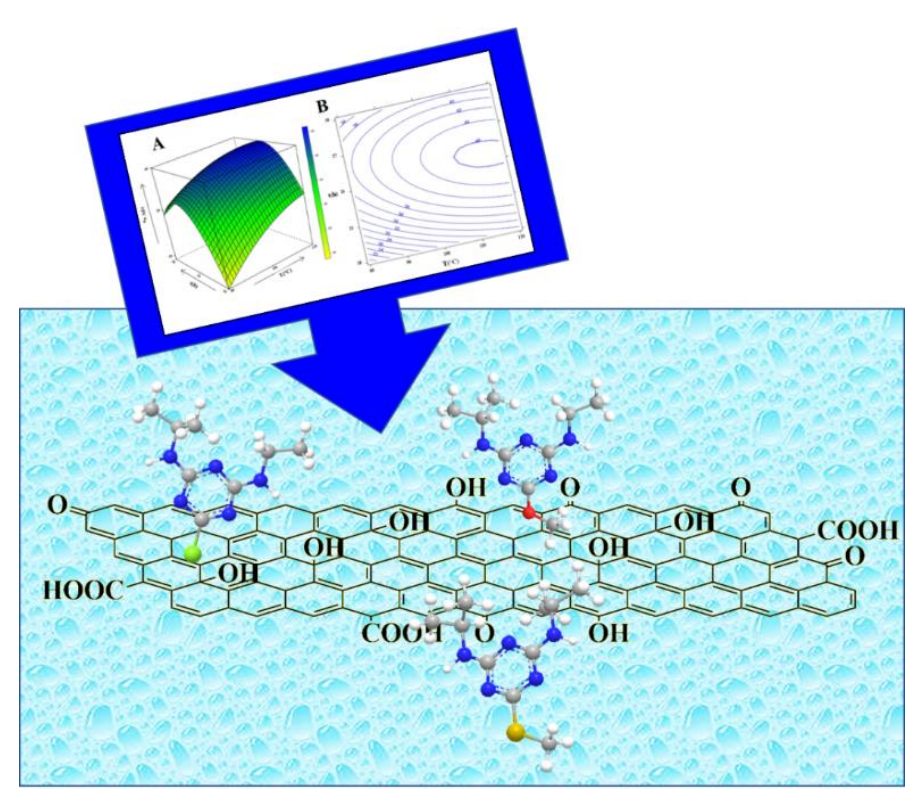

In this work, triazines were chosen as the organic micropollutants model, to develop a useful method for the removal of triazine products, using a reduced derivative of graphene oxide as adsorbent material. The pristine graphene oxide and its thermally reduced derivatives under mild conditions were tested, optimizing the GO reduction conditions by means of DOE coupled with the response surface methodology. For the reduction it was decided to choose the mildest and simplest conditions possible, using an air heat treatment in a common laboratory oven. The optimal reduction conditions deduced from the response surface were calculated at a reduction temperature of $110^{\circ} \mathrm{C}$ maintained for 24 hours and rGO sample was employed in the adsorption of the triazines. All the adsorbent materials have been characterized before use, by Scanning Electron Microscopy (SEM), X-ray diffraction (XRD), Fourier Transform Infrared Spectroscopy (FTIR), Xray photoelectron spectroscopy (XPS) and Brunauer-Emmett- Teller (BET) surface area analysis. Triazine analyses were performed by HPLC. The data obtained from the adsorption isotherms have been fitted with the Langmuir and Freundlich models, and the Freundlich model was the best one, especially for the Atraton and the Prometryn. The maximum adsorption capacity obtained was $4.4 \mathrm{mg} / \mathrm{g}$ for Atrazine, $19.4 \mathrm{mg} / \mathrm{g}$ for Atraton and $18.4 \mathrm{mg} / \mathrm{g}$ for Prometryn, at room temperature.

\section{Keywords}

reduced graphene oxide;

thermal mild reduction;

response surface methodology;

triazines removal;

Freundlich isotherm. 


\section{Introduction}

112 The adsorption of organic pollutants is one of the promising methodologies for their removal from the environmental,

113 and the interest towards efficient and low-cost materials for remediation of contaminants from water is strongly emerging 114 (Xiao and Pignatello 2015; Ruggieri et al. 2015; D'Archivio et al. 2018). Chemical oxidation, ion exchange, membrane 115 separation and adsorption have been widely applied for the removal of pollutants from water (Carolin et al. 2017; Jeevanantham et al. 2019). Among these, adsorption remains one of the most effective and important technologies, considering the different nature of the contaminants to be analysed, as well as being a versatile and economical method (Jung et al. 2001; Zhang et al. 2019; Mojiri et al. 2020).

119 One of the main causes of pollution of surface and groundwater is attributed to the increase in the use of herbicides in agricultural activities, causing great concern due to the potential risk to human health (Jablonowski, Schäffer, and Burauel 2011; Sousa et al. 2018).

Atrazine is an herbicide, of the triazine classes, typically used for the control of broadleaf season-long weeds in a variety of crops such as corn and sugarcane, but it also finds use on turfs such as golf courses and residential lawns as well (Frank and Sirons 1979; Miller et al. 2000). Human exposure to atrazine is linked to several serious health effects. A potent endocrine disrupter, atrazine interferes with hormonal activity of animals and humans at extremely low doses (Sanderson et al. 2002). It exhibits acute, chronic and phytotoxicity. It has been proved that atrazine contains mutagenic and carcinogenic agents also (MacLennan et al. 2002; Roberge, Hakk, and Larsen 2004; Kucka et al. 2012). Although the U.S. Environmental Protection Agency (USEPA) approved its continued use in October 2003, that same month the European Union (EU) announced a ban of atrazine because of ubiquitous and unpreventable water contamination (Bethsass and Colangelo 2006). USEPA set a Maximum Contaminant Level Goal (MCLG, i.e. the level 131 of a contaminant in drinking water below which there is no known or expected risk to health) for atrazine as $3 \mu \mathrm{g} / \mathrm{l}$, while 132 the recommended level of atrazine in drinking water in European Union countries is $0.1 \mu \mathrm{g} / \mathrm{l}$. An Atrazine Monitoring 133 Program (AMP) was created in the US to determine whether concentrations of atrazine and its chemical degradates are 134 present at a level that could potentially pose a risk to public health. In September 2020, USEPA released the Interim 135 Registration Review Decisions for atrazine, propazine, and simazine, and finalized mitigation measures to protect human 136 health and mitigate potential ecological risks.

137 Among the class of triazines, Atrazine, Atraton and Prometryn (shown in Figure 1) were chosen as a model for the study 138 of the removal of contaminants from water and the adsorbent material chosen was a reduced graphene oxide (rGO), whose 139 adsorbent capacity has been optimized. 
a)

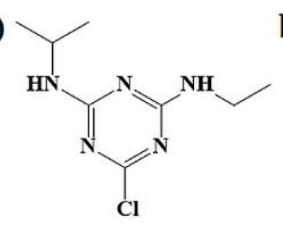

Atrazine

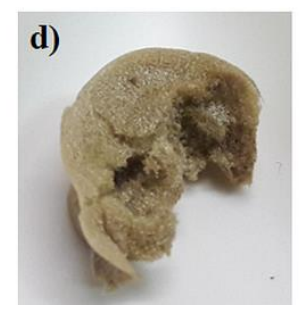

b)

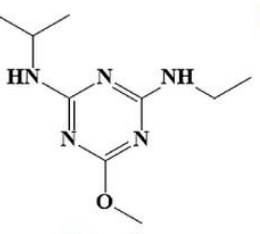

Atraton

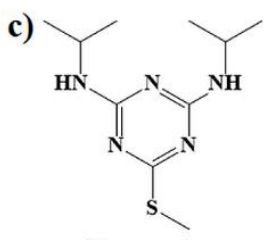

Prometryn

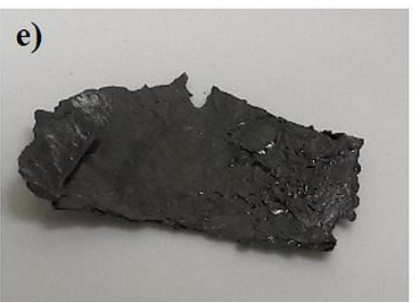

Figure 01 Triazines used in this work: a) Atrazine, b) Atraton and c) Prometryn; Graphenic materials used as sorbent:

d) pristine GO sponge obtained by freeze drying the aqueous dispersion and e) rGO film obtained by simple evaporation of the solvent in a petri dish.

Graphene-based materials are widely used for adsorbent removal of organic pollutants from water, due to their unique characteristics, including high adsorption capacity.

Graphene and its derivatives have attracted great attention all over the world for their potential applications in sensors, catalysis, energy-storage devices due to the excellent mechanical, electronic, and thermal properties (Geim and Novoselov 2007; Singh et al. 2011).

Many studies show that graphene-based materials have good potential in the environmental field (Thakur and Kandasubramanian 2019). For instance, their large surface area and delocalized $\pi$ network have been exploited in the adsorption of different types of contaminants from water (Lu and Astruc 2020). In general, an adsorbent material must have a good adsorption capacity which will depend on the surface properties of the adsorbent itself, on the presence of sites available to interact with the pollutants and on the ease of homogeneous dispersity in the aqueous media.

Design Of Experiment (DOE) coupled with the response surface methodology (RSM) has been wildly used in multiparametric optimization of analytical method conditions (Tarley et al. 2009; Vera Candioti et al. 2014; Ruggieri et al. 2017; Ruggieri et al. 2020) and pollutant removal methods, especially for the optimization of adsorption working conditions (temperature, $\mathrm{pH}$, time) or for the improvement of the synthesized materials (Lingamdinne et al. 2020; Bonetto et al. 2021). Most applications have involved GO-composite materials in order to modify and refine properties for a specific purpose. In detail, Central Composite Design was used to evaluate the adsorption mechanism and the influencing factors in the adsorption process of spilled oil by means of graphene/chitosan nanocomposite (Ghasemi et al. 2020). Tapouk et al. used the same multivariate approach to evaluate the potential, for the endotoxin removal, of GO sheet-LArginine nanocomposite (Amini et al. 2020). Three-factors Box-Behnken design was employed to optimise the synthesis 
of polymer-based nanocomposite (chitosan-polyethyleneimine-graphene oxide) for simultaneous removal of cationic and anionic heavy metal contaminants (Perez et al. 2017).

165 In this work, DOE coupled with RSM was chosen to optimize the graphene oxide reduction conditions in order to develop a useful method for the removal of triazine pesticides from the aqueous medium. The rGO material was synthesized from graphene oxide produced in the laboratory, after a mild heat treatment of the pristine GO.

Atrazine was chosen as the organic micropollutant model for the standardised batch adsorption tests and a three-level full factorial design was employed to plan the representative experiments. Eventually, the optimised sorbent material was tested on other triazine models namely Atraton and Prometryn. The idea behind the work was to couple an optimization step to the study of the triazine adsorption onto rGO; this was achieved by testing, according to the three-level full factorial design, graphene oxide derivatives thermally reduced. The objective was to use a simple and environmental friendly sorbent material that was optimised with a minimum number of experiments and in mild conditions, which are easily controllable and do not require an inert atmosphere. As far as our knowledge there are no studies that involve simple rGO films for atrazine adsorption.

Many groups used magnetic graphene oxide-based nanocomposites for the removal of pollutants for sustainable water purification. Zhao et al reported pioneering work on the use of a graphene-based $\mathrm{Fe} 3 \mathrm{O} 4$ magnetic nanoparticles as the adsorbent for the magnetic solid-phase extraction of some triazine herbicides in environmental water (Zhao et al. 2011) followed by high performance liquid chromatography.

Boruah et al. used $\mathrm{Fe} 3 \mathrm{O} 4 /$ reduced graphene oxide $(\mathrm{rGO})$ nano composite, which is easily and quickly separated from the aqueous medium using an external magnet for its reuse (Boruah et al. 2017).

Andrade et al. reported composite magnetic nanoparticles embedded into pristine GO sheets as adsorbents for the removal of atrazine, using organic solvent (ethanol) and an ultrasonic process (Andrade et al. 2019). atrazine and lead ions (Zhang et al. 2018). The material was synthetised via slow pyrolysis of graphene oxide pre-treated corn straws in a high temperature furnace at $600^{\circ} \mathrm{C}$ under nitrogen.

Pristine materials usually have lower capability for the removal of organic pollutants compared to hybrid or polymeric composite. Recently, Souza Antônio et al. described, in detail, the adsorption process involving atrazine, as a target substrate, and GO as sorbent material (de Souza Antonio et al. 2020). The study evaluated the changes in the adsorption capacity following the variation of $\mathrm{pH}$, concentration, temperature and dissolved salts. However, it seems that no experimental design was applied, limiting the system understanding when the multivariate nature of the adsorption phenomenon is not considered. Furthermore, no mention is made about the recoverability of the material, which is 
Differently from the described studies, in this work the blandest possible conditions have been chosen, the possibility to optimize the adsorption capability of an rGO film (easily recoverable material) was considered.

196 Reduced graphene oxide samples were prepared by mild thermal treatment of GO in laboratory oven, starting from acetone solutions of GO heat treated in air for $18-30$ hours at $80-120^{\circ} \mathrm{C}$, to obtain thick films of rGO.

Furthermore, the outcomes of the DOE model were exploited to better understand not only the adsorption process but also the effects that the reduction parameters have on the material properties. Even if the percentage of analyte adsorption does not achieve impressive values, the study could be surely a starting point for further works that could involve the optimization of the adsorption conditions.

\section{Materials and methods}

GO was prepared from graphite flakes with an average particle size of 100 meshes purchased from Sigma-Aldrich 99\%, product N. 221341), potassium permanganate (KMnO4, >99\%, product N. 60458), hydrogen peroxide solution ( $\mathrm{H} 2 \mathrm{O} 2,30 \%$, product N. 95294), hydrochloric acid ( $\mathrm{HCl}, 37 \%$, product N. 30721), Atrazine (product N. 45330, PeStANAL $®$ ), Atraton (product N. 31206, PESTANAL $($ ), Prometryn (product N. 45636, PeSTANAL®) and acetonitrile (product N. 34888, HPLC-grade Chromasolv ${ }^{\circledR}$ ) were purchased from Sigma Aldrich (St Louis, MO). All the aqueous solutions were prepared using MilliQ distilled water (Millipore, Bedford, MA, USA).

\subsection{GO/rGO preparation and characterization}

211 Graphene oxide was synthesized using a modified Hummers method (Treossi et al. 2009; Iacoboni et al. 2019). Graphite $212(5 \mathrm{~g})$, and sodium nitrate $(3.8 \mathrm{~g})$ were placed into a beaker in a salt/ice bath. Subsequently, $375 \mathrm{ml}$ of concentrated sulfuric 213 acid was added. The reaction mixture was kept in continuous agitation by a mechanical stirrer. After the mixture has 214 become homogeneous, $25 \mathrm{~g}$ of potassium permanganate were slowly added. The solution was kept under stirring for 5 215 days at room temperature. After 5 days a $5 \% \mathrm{H}_{2} \mathrm{SO}_{4}$ aqueous solution (700 ml) was poured through a funnel and $\mathrm{H}_{2} \mathrm{O}_{2}$ 216 (30 wt \%) was added drop by drop to remove the potassium permanganate and the suspension was thus stirred for another $2172 \mathrm{hr}$. In order to obtain a clean product, the mixture was diluted with $5 \% \mathrm{H}_{2} \mathrm{SO}_{4}(2 \mathrm{l})$ and left to settle for 1 day. Inorganic 218 impurities were removed through successive centrifuges, after removing the surnatant. The solid part was 219 washed/centrifuged at $4000 \mathrm{rpm}$ for 10 min with a 5\% aqueous solution of $\mathrm{H}_{2} \mathrm{SO}_{4}$ and $\mathrm{H}_{2} \mathrm{O}_{2}$ at $0.3 \%$ (12 times), then $4 \%$ $220 \mathrm{HCl}$ (3 times), deionized water (8 times), and finally MilliQ water (2 times), removing the supernatant after each passage. 221 The $\mathrm{pH}$ of the dispersion was monitored until it reached 6-7. Finally, the GO is transferred to acetone and dried at $50{ }^{\circ} \mathrm{C}$ for 24 hours, obtaining $4.8 \mathrm{~g}$ of powder. 
GO and rGO were fully characterized by Scanning Electron Microscopy (SEM), X-ray diffraction (XRD), Fourier

226 Transform Infrared Spectroscopy (FTIR), X-ray photoelectron spectroscopy (XPS) and Brunauer-Emmett-Teller (BET)

227 surface area analysis.

228 Surface topography was studied by Scanning Electron Microscope (SEM, Leo 1530 Gemini). The images were acquired 229 with an acceleration voltage of the electron beam, E.H.T. $=10 \mathrm{KV}$, at different magnifications. The GO sample for the 230 SEM was prepared by spin coating a very dilute aqueous solution $(0.2 \mathrm{mg} / \mathrm{ml}$, volume of 50 1) of the material on a silicon 231 substrate. The rGO film was deposited by drop casting a dispersion of the material in water on the Si substrate.

232 X-ray diffraction (XRD) analysis was made by a Panalytical X Pert Pro X-ray diffractometer on dry and pulverized 233 materials.

234 The Fourier transform infrared (FT-IR) spectra of GO/rGO were recorded on a FT-IR spectrometer (Perkin Elmer 235 spectrophotometer Spectrum Two) equipped with reflectance module (ATR). The samples were analysed directly in the 236 form of films.

237 X-ray photoelectron spectroscopy (XPS) spectra were collected in ultra-high vacuum (UHV) conditions with a PHI 1257 238 spectrometer, equipped with a monochromatic $\mathrm{Al} \mathrm{K \alpha}$ source $(\mathrm{h} \square=1486.6 \mathrm{eV}$ ) with a pass energy of $11.75 \mathrm{eV}$, 239 corresponding to an experimental resolution of $0.25 \mathrm{eV}$. The acquired XPS spectra have been fitted with Voigt line shapes 240 and Shirley backgrounds. The GO/rGO samples for the XPS were prepared by drop casting a dilute aqueous solution (1.0 $241 \mathrm{mg} / \mathrm{ml}$, volume of 50 1) of the material on a gold substrate.

242 BET isotherm adsorption measurements were performed by a nitrogen porosimeter (Quantachrome Instrument, 2008). 243 The device is controlled by the NOVA Series Windows®-Based Operating and Data Analysis Software. The 244 measurements were performed on dry and pulverized materials.

\section{$245 \quad 2.2$ Chromatographic analysis}

246 The analysis of the triazines was carried out by using an HPLC apparatus consisting of a controller pump Waters 600 247 equipped with on-line degasser Agilent Technologies 1220 series (Agilent Technologies, Waldbronn, Germany), an 248 autosampler Water 717 plus, a Security Guard Ultra Cartridge UHPLC C18 precolumn (4.6 mm id) from Phenomenex 249 (Torrance, CA, USA), a Kinetex XB-C18 (Phenomenex) column (250 mm length, $4.6 \mathrm{~mm}$ id, $5 \mu \mathrm{m}$ particle size) and a 250996 photodiode array detector (Waters). The working wavelengths for quantitative analysis of each analyte were $220 \mathrm{~nm}$. 251 The elution was performed at room temperature, constant flowrate $(1 \mathrm{ml} / \mathrm{min})$ and isocratic conditions using a mixture 252 (35:65, v/v) of water and acetonitrile. The chromatographic apparatus was controlled by Empower software (Waters). 253 The analysed solutions were filtered by HPLC filters Whatman Spartan13/02 RC.

\section{$254 \quad 2.3$ Adsorption experiments}


The batch triazines adsorption experiments were carried out at room temperature under shaker conditions. Ten milligrams of rGO film were placed in contact with $10 \mathrm{ml}$ of ultrapure water, in screw-cap glass vials, containing a single triazine. Sorption isotherm experiments were conducted with seven initial pesticide concentrations $(0.5,1.0,2.0,5.0,10,20$, and $50 \mu \mathrm{g} / \mathrm{ml})$. The point at the concentration of $10 \mu \mathrm{g} / \mathrm{ml}$ was repeated in triplicate. The vials of the nine samples containing different concentrations of pesticide were simultaneously placed on orbital shaker at $300 \mathrm{rpm}$ in the dark for $1 \mathrm{~h}$. After reaching equilibrium, $1 \mathrm{ml}$ of solution was collected, filtered with $0.2 \mu \mathrm{m}$ PTFE filters (PHENEX, Phenomenex) and placed in HPLC vials to determine the equilibrium concentration $\left(\mathrm{C}_{\mathrm{eq}}\right)$. Preliminary kinetic tests were achieved, and equilibrium was assumed when no further change in pesticide up-take was observed. Based on these experiments $1 \mathrm{~h}$ contact time was sufficient to reach equilibrium.

The adsorptions data can be understood using several approaches. The models usually applied are the Freundlich and Langmuir isotherms (Freundlich 1906; Langmuir 1916). The Freundlich isotherm [Equation (1)], is generally used to model the removal of hydrophobic organic pollutants from water. It is an empirical equation used to define the uptake of adsorbate occurring on a heterogeneous surface by multilayer adsorption:

$$
q_{e}=K_{F} C_{e}^{1 / n}
$$

where $\mathrm{q}_{\mathrm{e}}$ is the equilibrium adsorbate concentration onto the adsorbent, $\mathrm{C}_{\mathrm{e}}$ is the adsorbate equilibrium concentration in the solvent, $\mathrm{K}_{\mathrm{F}}$ (Freundlich constant) indicates the multilayer adsorption capacity of adsorbent and 1/n measures the adsorption intensity or surface heterogeneity of the adsorbent. It becomes more heterogeneous as gets closer to zero, and homogeneous if this value approach to one (D'Archivio et al. 2009; Berhane et al. 2017; Nodeh et al. 2019). The amount of analytes adsorbed onto the adsorbent after equilibration [Equation (2)] was established by mass balance of the process at equilibrium condition:

$$
q_{e}=V \frac{\left(C_{0}-C_{e}\right)}{m}
$$

where $\mathrm{C}_{0}$ is the initial concentration, $\mathrm{m}$ is the mass of adsorbent and $\mathrm{V}$ is the solution volume. The Langmuir model [Equation (3)] assumes uniform energy sites on the adsorbent surface and is defined by the following relationship:

$$
q_{e}=\frac{q_{\max } K_{L} C_{e}}{1+K_{L} C_{e}}
$$

where $\mathrm{q}_{\max }$ is the limiting amount of adsorbate per unit of adsorbent required for a monolayer coverage of adsorbent surface and KL, the Langmuir adsorption constant is a binding constant related to the free energy of sorption. The reciprocal value of KL corresponds to the concentration in the liquid phase at which half of the maximum adsorption capacity of the adsorbent is reached. The isotherm adsorption data can be described in the following linear forms of Freundlich [Equation (4)] and Langmuir [Equation (5)] models, respectively:

$$
\log q_{e}=\log K_{F}+\frac{1}{n} \log C_{e} \text { (4) }
$$




$$
\frac{C_{e}}{q_{e}}=\frac{1}{q_{\max } K_{L}}+\frac{C_{e}}{q_{\max }}
$$

thus, the model parameters in both cases can be easily obtained from least-square linear regression of the experimental data.

\subsection{Response surface methodology}

Response surface methodology (RSM) is a chemometric tool commonly used to graphically identify an optimum, that is the point (maximum or minimum in the experimental domain) at which the combination of the experimental variables results in the best response (Lundstedt et al. 1998). For optimization purpose, it is crucial to plan the experiments according to an appropriate experimental design to well describe the curvature of the quadratic model. A three-level full factorial design is frequently coupled with RSM since it ensures acceptable reliability in estimating individual and combined effects of the independent variables on the response (Vander Heyden et al. 2001). Thus, the relationship between the response and the factors can be well approximated, in the limited domain, by a second-order polynomial function [Equation (6)]:

$$
\mathrm{Y}=a_{0}+a_{10} X_{1}+a_{2} X_{2}+a_{12} X_{1} X_{2}+a_{11} X_{1}^{2}+a_{11} X_{2}^{2}
$$

where $\mathrm{Y}$ is the response, $\mathrm{X}_{\mathrm{i}}$ the experimental variables and $\mathrm{a}_{\mathrm{i}}$ the regression coefficients. Determining the model coefficients by ordinary least squares regression, the value of the response $Y$ can be computed in each point of the explored domain and can be plotted in a three-dimensional Response Surface, providing easier exploitation of the interesting information. The RSM and the three-level full factorial design were applied to assess the influence of the Temperature (T) and the time (t) of the thermal treatment, performed for GO reduction, on the rGO film adsorption efficiency.

Factors and levels were defined considering previous knowledge and preliminary outcomes. The experiments, reported in Table 01, were performed in random order and consisted of the nine best variable combinations and one replicate in the central point. The optimal experimental condition was selected maximizing the adsorption percentage resulting from standardized batch adsorption experiments, which were carried out keeping constant the amount of rGO, the triazine and its concentration (10 $\mathrm{mg}$ of $\mathrm{rGO}$ in $10 \mathrm{ml}$ of atrazine aqueous solution at a concentration of $5 \mathrm{~g} / \mathrm{ml}$ ). Analysis of variance (ANOVA) was performed to statistically identify the influencing factors, to evaluate the significance of the model and the lack-of-fit. The determination coefficient, the related adjusted value ( $\mathrm{R}^{2}$ and $\mathrm{R}_{\text {adj }}$ ) as well as the 311 and generalization level. The statistical analysis was performed using the R-based free software "Chemometric Agile 312 Tool" (CAT, Chemometric Agile Tool, Leardi, R. et al 2019; http://gruppochemiometria.it/index.php/software).

\section{$313 \quad 3$ Results}

\section{3.1 Preparation and characterization of the sorbents}


315 This work was carried out starting from a graphene oxide produced by us in the laboratory, following a protocol already extensively studied in the literature (Treossi et al. 2009) and already described in our other works. Since the starting material used is not a commercial product, we have reported the characterization of the graphene oxide from which we started (see the Support Information), and the chemical characterization of the material itself.

319 In the Supporting Information we reported the GO characterization by Scanning Electron Microscopy (SEM), Fourier 320 Transform Infrared Spectroscopy (FTIR) and X-ray photoelectron spectroscopy (XPS).

321 For SEM images, the starting aqueous GO solution at a concentration of $0.2 \mathrm{mg} / \mathrm{ml}$ was spin coated on a silicon substrate, and showed a typical dispersion of graphene oxide sheets (Figure S01 a-b), constituted of mono and multilayers, whose lateral dimensions ranged from $100 \mathrm{~nm}$ up to $100 \mathrm{~m}$. The GO showed the presence of some characteristic folds and ripples.

The GO FT infrared spectrum showed in Supporting Information (Figure S02) evidenced the -OH stretching vibration at about $3420 \mathrm{~cm}^{-1}$. The vibrational bands at $2923 \mathrm{~cm}^{-1}$ and $2854 \mathrm{~cm}^{-1}$ are attributed to $-\mathrm{CH}_{2}$. The absorption band at 1725 $\mathrm{cm}^{-1}$ corresponds to stretching vibrations of $\mathrm{C}=\mathrm{O}$ from carbonyl or conjugated carbonyl groups. The absorption peak at $1620 \mathrm{~cm}^{-1}$ is assigned to the $\mathrm{C}=\mathrm{C}$ (aromatics) stretching. The absorption peaks at about $1423 \mathrm{~cm}^{-1}, 1225 \mathrm{~cm}^{-1}$ and 1060 $\mathrm{cm}^{-1}$ are assigned to -OH from carboxyl, C-O-C from epoxy or ether and C-O from alkoxy, respectively. These results are in agreement with the literature (Iacoboni et al. 2019).

In Figure S03 we showed the XPS survey of the graphene oxide (A) and the area corresponding to the C 1s signal (B), from which we can observe the presence of a high percentage of oxygenated groups in the GO. From XPS analysis we calculated the $\mathrm{C} / \mathrm{O}$ ratio that was 1.99 . The relative area percentage for $\mathrm{C}-\mathrm{C}, \mathrm{C}-\mathrm{O}$ and $\mathrm{C}=\mathrm{O}$ were 44.0, 51.5, 4.5 (as reported in Table 02), which confirms the presence of a high number of oxygenated groups in the starting sample. Going into detail, we can see the contributions of the hydroxyl and epoxy groups on the carbonaceous skeleton, which make the peak relative to the C-O very intense. The XPS C 1s core level spectra are displayed in Figure S03-B. The spectrum was fitted by the sum of three components assigned to $\mathrm{C}$ atoms belonging to: aromatic rings and hydrogenated carbon $(\mathrm{C}=\mathrm{C} / \mathrm{C}$ C, $284.8 \mathrm{eV})$, hydroxyl and epoxy groups (C-O/C-O-C, $286.9 \mathrm{eV})$ and carbonyl groups $(\mathrm{C}=\mathrm{O}, 288.2 \mathrm{eV})$. The relative abundances of each component of the $\mathrm{C} 1 \mathrm{~s}$ spectra are: $\mathrm{C}=\mathrm{C} / \mathrm{C}-\mathrm{C} 44.7 \%, \mathrm{C}-\mathrm{O} / \mathrm{C}-\mathrm{O}-\mathrm{C} 47.7 \%, \mathrm{C}=\mathrm{O} 7.6 \%$. The resulting quantitative estimate of the $\mathrm{C} / \mathrm{O}$ concentration ratio is 1.99 , showing a high degree of oxidation of the material (this ratio

342 The presence of a well oxidized starting material can influence the subsequent thermal reduction that has been chosen. In 343 this work we decided to work in mild reduction conditions, using a simple laboratory oven and carrying out the reduction 344 in air. The choice of such simple and easily replicable conditions in any laboratory, without having to use more 
sophisticated and expensive equipment, was made with the aim of being able to easily obtain a reduced material that does not disperse in the aqueous phase and could therefore be easily separated. from the solution and recovered.

When working in these mild conditions it is important to have an indication of the degree of oxidation of the starting material, because it has already been seen in the literature that by submitting graphene oxide to reduction in air, up to 100-120 ${ }^{\circ} \mathrm{C}$ extreme degradation of the material and a loss of carbon as amorphous or carbon dioxide are not expected, processes that occur at higher temperatures (Perrozzi et al. 2014).

From studies previously carried out on samples reduced in mild conditions it was found that at $80-120^{\circ} \mathrm{C}$ the thermal reduction of a graphene oxide layer led to the loss of water of hydration and of the more labile groups present on the graphene skeleton, i.e. the epoxy groups (Catanesi et al. 2018).

In this work, the adsorption of atrazine was carried out starting from the pristine GO material and on some of its thermally reduced derivatives at a temperature between 80 and $120{ }^{\circ} \mathrm{C}$, and considering a reduction time varying between 18 and 30 hours total.

To achieve the maximum adsorption capacity, the best reduction conditions were determined to obtain an optimal sorbent. An experimental multivariate design with two independent variables, time $(t)$ and temperature $(T)$, was used. For each independent variable, three different levels were considered. Each sample was used to evaluate the different adsorption capacity through batch tests. Response surface methodology (RSM) was used for the optimizations of experimental variables.

Before going into the details of the methodology chosen, we asked ourselves which form of the solid material was the most suitable in our case. Indeed, the synthesized GO and rGO could be used in two different 3D forms with different chemical-physical properties: the thick film and the sponge. To obtain the film, the graphenic material was placed in a crystallizer with acetone and dried. The sponge, on the other hand, was obtained after a freeze-drying process which allows the elimination of water from an iced water solution by sublimation. The sample was frozen at a temperature of about $-20{ }^{\circ} \mathrm{C}$ and brought to low pressure through a rotary pump. As the temperature of the sample increased, the ice was sublimed, obtaining a three-dimensional sponge. Both processes were simple, with the least possible deterioration of the structure and components of the substance itself.

As already mentioned, the materials have very different chemical-physical and structural characteristics. The threedimensional sponge has a higher adsorption capacity but with a high contact time, it is easily dispersed in an aqueous solution. The thick film shows a lower adsorption capacity than the sponge, but allows easy recovery, as it does not disperse in solution. In Figure 1 the two forms are shown. An experimental evidence of the material reduction can be seen in Figure 1, where the colour change of the material reduced from a pale brown (GO sponge) into dark black is evident (rGO film). 
Preliminary adsorption tests were conducted initially using an aqueous solution of pesticides and graphene oxide. Due to its poor hydrophobicity, the total solubilization of graphene oxide in aqueous solution has occurred, both in the form of thick film and sponge, simultaneously showing the poor adsorption capacity against pesticides. Subsequently, the material was thermally reduced (rGO) increasing its hydrophobicity and adsorption capacity.

The two forms of rGO, sponge and film, were therefore compared. For reasons related to a possible recovery of the material, the rGO thick film was chosen. In fact, the rGO sponge still showed a redispersion behaviour in the aqueous phase, effectively preventing the separation of it from the aqueous phase.

383 The reduction of graphene oxide, previously synthesized, took place thermally in the air. The GO samples were dispersed 384 in acetone, placed in a petri dish and heat treated in air for 18-24-30 hours in a laboratory oven, to obtain a well-adhered uniform film of reduced $\mathrm{GO}(\mathrm{rGO})$. The reduction temperature was chosen between 80 and $120{ }^{\circ} \mathrm{C}$, and subsequently the samples were used for preliminary measurements of adsorption with atrazine, optimizing the conditions by means of an experimental design.

DOE-RSM was employed to evaluate the influence of temperature and time and their combined effects on the adsorption efficiency of a thermally reduced GO film. The selected DOE consists of three levels for both Temperature (80, 100 and $\left.120^{\circ} \mathrm{C}\right)$ and time $(18,24$ and $30 \mathrm{~h})$; the resulting experimental data were regressed with the reported equation [Equation (7)] providing the following model:

393 where the standard deviations of the coefficients are given in parenthesis.

394 All the linear terms show relevant effects (significance level of 5\%) whereas, except for $\mathrm{t}^{2}$, the other quadratic terms are not significant. The observed and the computed response values (\%abs), for each point of the DOE, are displayed in Table 396 01; a good agreement is demonstrated between the calculated \% abs values and the experimental data with residuals randomly distributed. The surface model exhibits a satisfactory descriptive and predictive performance as witnessed by 398 the determination coefficient $\mathrm{R}^{2}(0.963)$, the related adjusted value (0.857) and the determination coefficient in leave399 one-out cross-validation (0.793). Moreover, the reported results of the ANOVA (Table 01) reveal that the surface model 400 is highly significant, according to the p-value $<0.05$, and that well fits the experimental data, since the lack-of-fit p-value 401 is greater than the significance level of $5 \%$. 

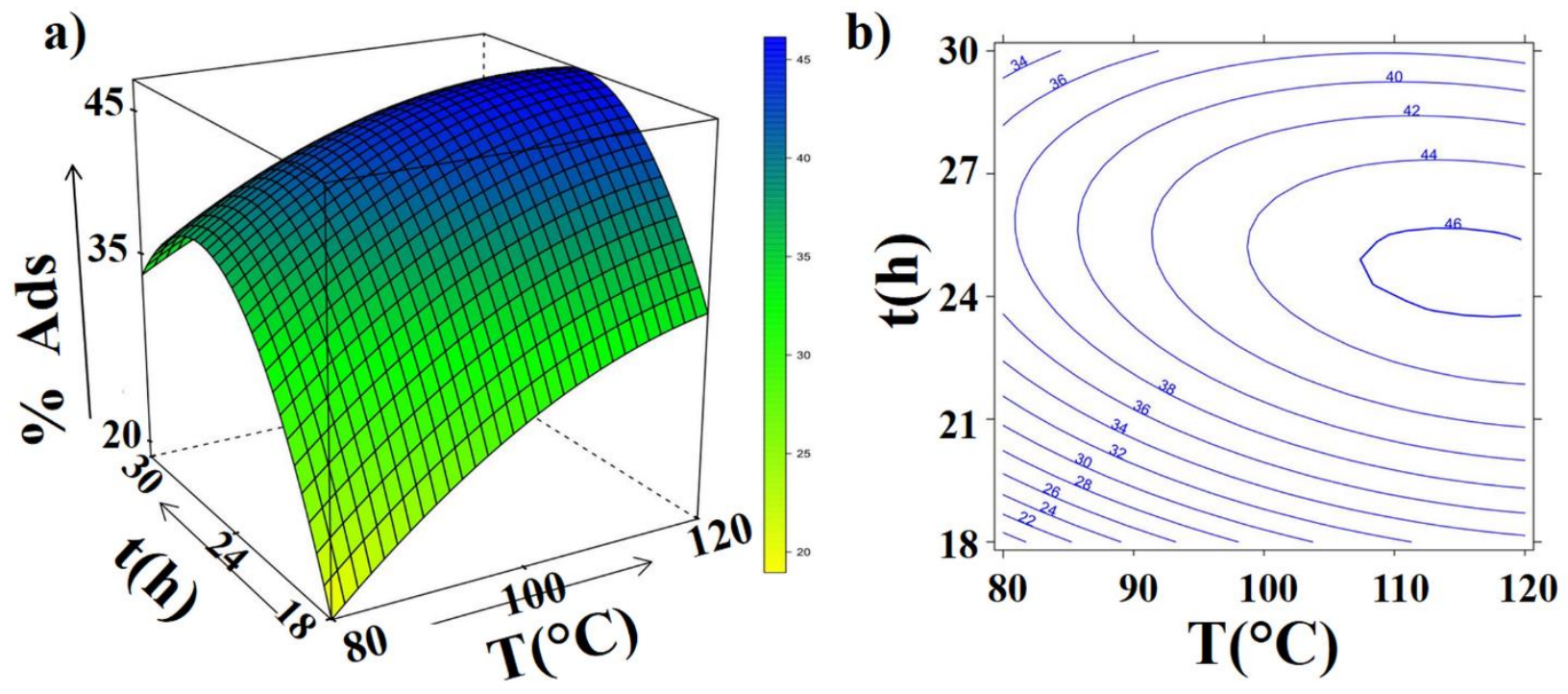

Figure 02 Surface plot $\mathbf{a}$ ) and related contour plot $\mathbf{b}$ ) of the percentage of adsorbed atrazine as a function of the reduction process parameters (temperature and time).

Figure 02 displays the response surface and the related iso-response plot. It can be noted that the maximum response computed by the model does not exceed the $46 \%$ of adsorption and that no improvement can be achieved by working in a temperature range between 110 and $120^{\circ} \mathrm{C}$ when the time is fixed at $24 \mathrm{~h}$. Since the maximum is at the extremity of the experimental domain and that no improvement is achieved by moving from $110^{\circ} \mathrm{C}$ to $120^{\circ} \mathrm{C}$ it was chosen to work under the mildest possible conditions. Accordingly, the thermal reduction was conducted with the following optimal working conditions: $\mathrm{T}=110^{\circ} \mathrm{C}, \mathrm{t}=24 \mathrm{~h}$. Furthermore, by integrating the DOE-RSM model outcomes and the information provided

411 by the characterization of the optimal rGO film, a better understanding of the system involved in the adsorption process 412 could be obtained. DOE-RSM allows to identify the influencing factors and to evaluate the effect of the process parameters on the rGO film adsorption ability. In this respect, a direct interpretation of the effect of temperature can be 414 done since the interaction term ( $\mathrm{Tt}$ ) and the quadratic one have resulted statistically not significant. In detail, an improvement of the \%abs can be obtained by increasing the temperature from $80^{\circ}$ to $120^{\circ} \mathrm{C}$ with an averaged effect on the response of near ten percentage points.

In general, the adsorption of organic pollutants is increased with the reduction of the GO in which the functional groups al. 2013).

420 On the other hand, a quadratic trend can be confirmed as regards the time dependence of the response, with the maximum 421 pinpointed at $\mathrm{t}=24 \mathrm{~h}$. After 24 hours of thermal treatment, the product reached the maximum efficiency of the mild reduction, which mainly concerned the more labile oxygenated groups present on the skeleton of the graphenic material, 
that is the epoxides and hydroxides, as confirmed by the FTIR analysis in which it is seen the decrease of the characteristic peaks. The aromatic skeleton as well as the carboxyl, carbonyl, epoxy and hydroxy groups on rGO were the major sites of adsorption, and there are interactions and hydrogen bond interactions of rGO with atrazine.

\begin{tabular}{lcccc}
\hline Parameters & Value $\pm \mathrm{SD}$ & $\mathrm{R}^{2}$ & Adj-R $^{2}$ & $\mathrm{Q}^{2}$ \\
\hline intercept & $44 \pm 2$ & & & \\
$* \mathrm{X}_{1}$ & $4.7 \pm 1.3$ & & & \\
$* \mathrm{X}_{2}$ & $4.3 \pm 1.3$ & 0.936 & 0.857 & 0.793 \\
$\mathrm{X}_{1} \cdot \mathrm{X}_{2}$ & $-2.5 \pm 1.6$ & & & \\
$\mathrm{X}_{1}{ }^{2}$ & $-2 \pm 2$ & & & \\
$* * \mathrm{X}_{2}{ }^{2}$ & $-11 \pm 2$ & & \\
\hline
\end{tabular}

\begin{tabular}{|c|c|c|c|c|c|}
\hline $\begin{array}{l}\text { Variation } \\
\text { source }\end{array}$ & $\begin{array}{l}\text { Sum of } \\
\text { Squares }\end{array}$ & $\begin{array}{l}\text { Degrees of } \\
\text { freedom }\end{array}$ & Mean Square & F-value & $p$-value \\
\hline Lack of fit & 35 & 3 & 11.7 & 2.4 & 0,43 \\
\hline Pure error & 4.8 & 1 & 4.8 & & \\
\hline Model & 589.4 & 5 & 117.9 & 11.8 & 0,02 \\
\hline Residual & 39.8 & 4 & 9.9 & & \\
\hline
\end{tabular}

Table 01 Model adsorption parameters and analysis of variance

Table 01 shows the determination coefficients $\left(\mathrm{R}^{2}, \operatorname{Adj}-\mathrm{R}^{2}, \mathrm{Q}^{2}\right)$, the model parameters with the corresponding significance level expressed by stars $\left({ }^{*} \mathrm{p}<0.05,{ }^{*} \mathrm{p}>0.01\right)$, the regression coefficients with the related standard deviation $(\mathrm{SD})$ and the Analysis Of Variance (ANOVA).

\section{$430 \quad 3.2$ Characterization of reduced Graphene Oxide (rGO) at $110^{\circ} \mathrm{C}$}

431 From the response surface obtained, the optimal reduction conditions of the material were deduced which are $110^{\circ} \mathrm{C}$ for 432 a time of 24h. rGO samples were fully characterized by Scanning Electron Microscopy (SEM), X-ray diffraction (XRD), 433 Fourier Transform Infrared Spectroscopy (FTIR), X-ray photoelectron spectroscopy (XPS) and Brunauer-Emmett-Teller 434 (BET) surface area method.

435 All the reduced materials have been characterized but only the characterizations relating to the reduced material at $110^{\circ}$ 436 C for a time of 24 hours are reported.

437 Surface topography of the rGO film showed homogeneous morphology, as evidenced in the Figure 03 a-c, where has 438 been reported the $\mathrm{rGO}$ film reduced at $110^{\circ} \mathrm{C}$. In the enlarged images on the right (b-c) you can see some wrinkles and folds on the surface of rGO films. 
440 The XRD patterns of GO shown in Figure 03-d (black line) reveal a GO diffraction peak at $2 \theta=11.1^{\circ}$, which implies an 441 interplanar space of about $0.81 \mathrm{~nm}$ (calculated by Bragg equation), corresponding at the reflection plane (001). In the 442 rGO sample the GO signal is almost completely absent and a broader signal at $2=23.9^{\circ}$ is due to the presence of a 443 graphitic skeleton (graphite XRD pattern shows a single, very intense and sharp peak at $26.8^{\circ}$ ) obtained after thermal 444 reduction of the oxygenated functional groups on the graphene oxide sheets. The peak becomes wider and less intense 445 and the interplanar space of rGO is reduced to $0.35 \mathrm{~nm}$.

446 The Fourier Transform Infrared (FTIR) analysis was performed in the range of wavenumbers of $4000-400 \mathrm{~cm}^{-1}$ for the 447 identification of functional groups. Analysing the rGO spectra in comparison with the starting GO we observed the 448 presence of different absorption peaks, according to the spectra reported in the literature (Figure 03-e).

449 In the $110^{\circ} \mathrm{C}$ reduced GO spectrum, we saw that the absorption peaks at $1423 \mathrm{~cm}^{-1}$ (-OH stretching vibrations from 450 carboxyl) in the curve of GO disappeared, and the relative intensity of C-O-C peak at $1225 \mathrm{~cm}^{-1}$ and $\mathrm{C}-\mathrm{O}$ at $1060 \mathrm{~cm}^{-1}$ 451 were lowered, the mild reduction of the sample.

452 FTIR analysis confirms the occurrence of the reduction process, which was not much efficient at those heating 453 temperature conditions, leaving the most of oxygenated functionality on the graphenic skeleton. 

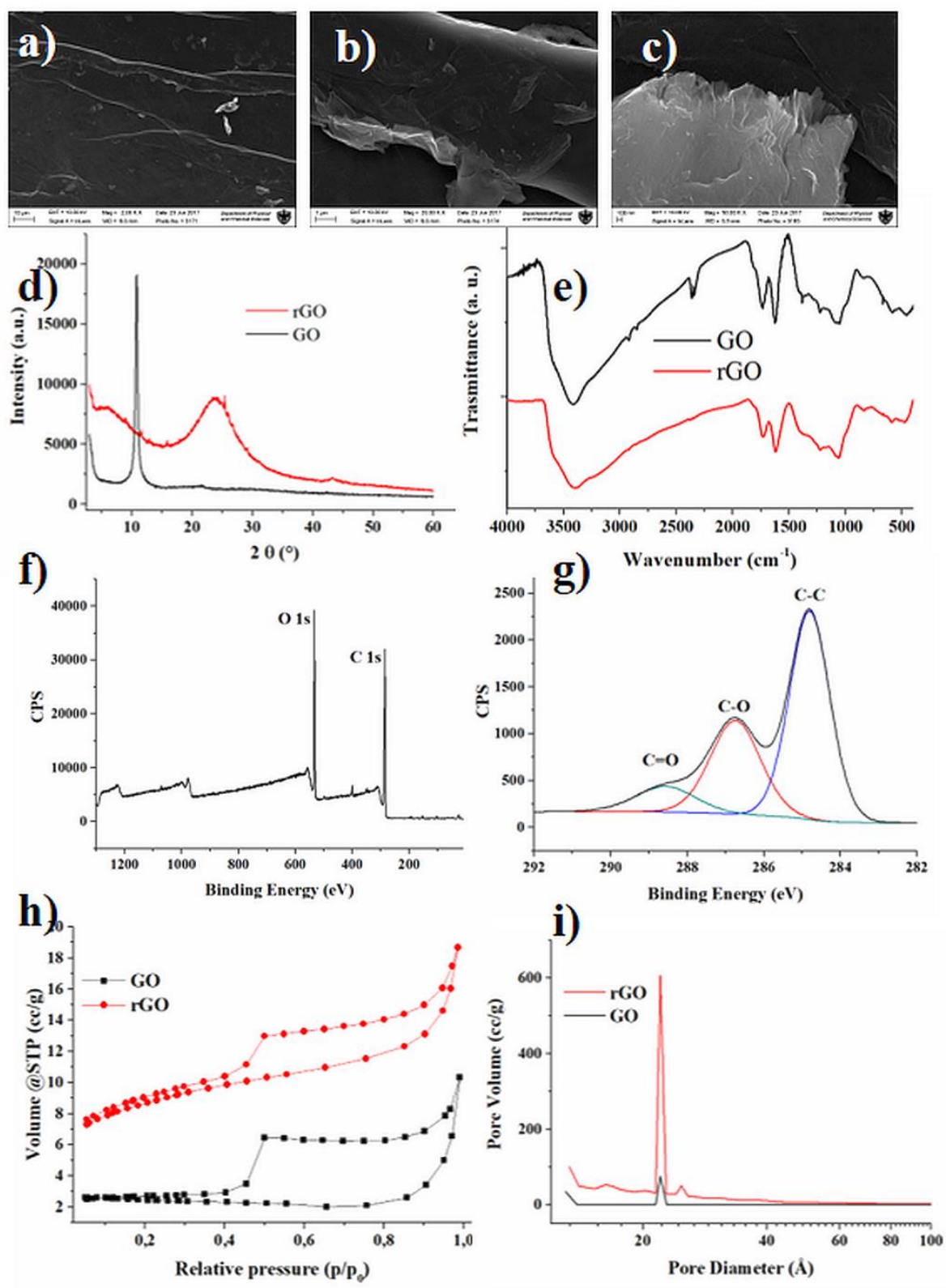

455 Figure 03 a-c) SEM images of rGO film reduced at $110^{\circ} \mathrm{C}$; d) XRD patterns of GO (black line) and rGO film reduced 456 at $110{ }^{\circ} \mathrm{C}$ (red line); e) FTIR spectra of GO and reduced GO at $110^{\circ} \mathrm{C}$; XPS survey of $\mathrm{rGO}$ at $110{ }^{\circ} \mathrm{C}(\mathbf{f})$ and $\mathrm{C} 1 \mathrm{~s}$ region

(g); BET isotherm (h) for GO (black square) and rGO (red circle) and (i) BJH pores average volume and diameter for GO (black line) and rGO (red line)

459 XPS was employed to study the chemical states of the prepared GO and rGO film. From XPS survey spectra (Figure 03-

460 f) we calculated the total content (\%) of $\mathrm{C} 1 \mathrm{~s}$ and $\mathrm{O} 1 \mathrm{~s}$ peaks, and the $\mathrm{C} / \mathrm{O}$ ratio calculated was respectively 1.99 for GO 461 and 2.51 for reduced $\mathrm{GO}$ at $110{ }^{\circ} \mathrm{C}$ respectively (see Table 02 ). In the $110^{\circ} \mathrm{C}$ reduced $\mathrm{rGO}$ we found an increasing in the $462 \mathrm{C} / \mathrm{O}$ ratio, as expected for reduced samples, due preferably to cleavage of $\mathrm{C}-\mathrm{O}-\mathrm{C}$ bond in the epoxy groups and $\mathrm{C}(=\mathrm{O})-$ $\mathrm{OH}$ from carboxyl.

\section{XPS survey}




\begin{tabular}{|l|c|c|c|}
\hline & C 1s (\%) & O 1s (\%) & C/O ratio \\
\hline GO & 64.6 & 32.4 & 1.99 \\
\hline rGO @ 110 & & & \\
& 68.7 & 27.4 & 2.51 \\
\hline \multicolumn{4}{|c|}{ C 1s fitting } \\
\hline & \multicolumn{2}{|c|}{ Relative area percentage (\%) } \\
\hline & C-C & C-O & C=O \\
\hline GO & 54.9 & 34.3 & 10.8 \\
\hline rGO @ $\mathbf{~ 1 1 0 ~}^{\circ} \mathbf{C}$ & 57.4 & 32.1 & 10.5 \\
\hline
\end{tabular}

Table 02 XPS survey: atomic percentages of $\mathrm{C}, \mathrm{O}$ for GO and rGO sample; Analysis of the deconvoluted C1s peaks obtained from XPS and relative area percentages for GO and rGO sample.

In Figure $03-\mathrm{g}$ are reported the $\mathrm{C} 1 \mathrm{~s}$ comparison spectra of the samples. The $\mathrm{C} 1 \mathrm{~s}$ core level spectra were fitted by summing three components assigned respectively to aromatic sp2 carbon (C-C), epoxy and hydroxyl groups (C-O) and carbonyl and carboxyl groups $(\mathrm{C}=\mathrm{O})$. From the analysis of deconvoluted peaks, we noticed an increase from $54.9 \%$ to $57.4 \%$ of the C-C contribution, while the C-O signal decrease from $34.3 \%$ to $32.1 \%$ in the $110{ }^{\circ} \mathrm{C}$ reduced sample. For the rGO the peak contributes at about $284.5 \mathrm{eV}$ relative to $\mathrm{C}-\mathrm{C}$ signal increased while the peak at about $286.5 \mathrm{eV}$ (C-O bond) becomes broader and reduced in intensity. This confirms the (partly) reduction of graphene oxide to graphene-like sheets by removing the oxygen-containing groups with the recovery of a conjugated structure. The peak relative to the $\mathrm{C}=\mathrm{O}$ double bond is superimposed on the peak relative to the C-O signal, and its contribution is difficult to deconvolve.

In table 02 are shown, in details, the relative percentage of deconvoluted $\mathrm{C}$ 1s peaks contribute to the GO and rGO samples.

Through the adsorption of nitrogen gas it was possible to evaluate the adsorption capacity, the surface area and verify the presence and size of the pores in the rGO film. The study of the specific volume of adsorbed nitrogen allows to determine the specific surface area of the materials, the specific volume and the diameter of the pores. The specific surface of a solid is the surface area per unit of mass and is expressed in $\mathrm{m}^{2} / \mathrm{g}$ and is determined with the Brauner, Emmet and Teller equation, or more simply BET method.

In Figure 03-h it is shown the GO and rGO adsorption and desorption isotherm, in which the presence of a moderate hysteresis phenomenon is visible, more evident for GO. The isotherm has a convex shape, classified as III type, representative of weak adsorbent-adsorbate interactions. A classification of pores is given by the International Union of Pure and Applied Chemistry (IUPAC) which classifies them according to their size and defines: micropores with a width below $2 \mathrm{~nm}$, mesopores with a width between 2 and $50 \mathrm{~nm}$ and macropores with a width greater than $50 \mathrm{~nm}$. 
Mesopores with an average diameter of $3 \mathrm{~nm}(30 \AA)$ were calculated, and loop of $\mathrm{H} 3$ type are found in both materials (GO and $\mathrm{rGO}$ ), mostly associated with the pore shape of solids consisting of aggregated non-rigid plate-like particles (Bardestani, Patience, and Kaliaguine 2019).

The hysteresis loop can be explained by the fact that since it is a thick film obtained by evaporation of the solvent, therefore of a not real porous material, the channels may not be completely open, this implies a different path of the gas between the adsorption and desorption phase. The measured specific surface area of the rGO sample was approximately $30 \mathrm{~m}^{2} / \mathrm{g}$, while for GO was approximately $8 \mathrm{~m}^{2} / \mathrm{g}$.

The specific surface area of the reduced samples is lower than the theoretical monolayer graphene oxide reported in literature which ranged from 2-1000 $\mathrm{m}^{2} / \mathrm{g}$ (Zhang et al. 2020), potentially due to the aggregation of the graphenic sheets which can cause their partial overlap and coalescence, especially the smaller ones, lowering the surface area of the material.

However, the presence of a crumpled three-dimensional structure of the sheets still leaves many exposed surface areas.

498 With the BJH numerical integration method (Barrett, Joyner, Halenda) the pores average volume and the average diameter were assessed both in the adsorption phase and in the desorption phase (Figure 03-i). From the data obtained, mesopores with an average diameter of $3 \mathrm{~nm}(30 \AA)$ and an average volume of $0.023 \mathrm{~cm}^{3} / \mathrm{g}$ are found both in the adsorption phase and in the desorption phase of rGO.

\subsection{Adsorption isotherm}

The adsorption of the triazines on the rGO film is studied using the linear form of Freundlich and Langmuir models (equations 4 and 5). Figure 04-a displays the observed adsorption equilibrium data on rGO, fitted with the Langmuir model, while Fig. 04-b shows the data of three herbicides interpolated with the Freundlich model.
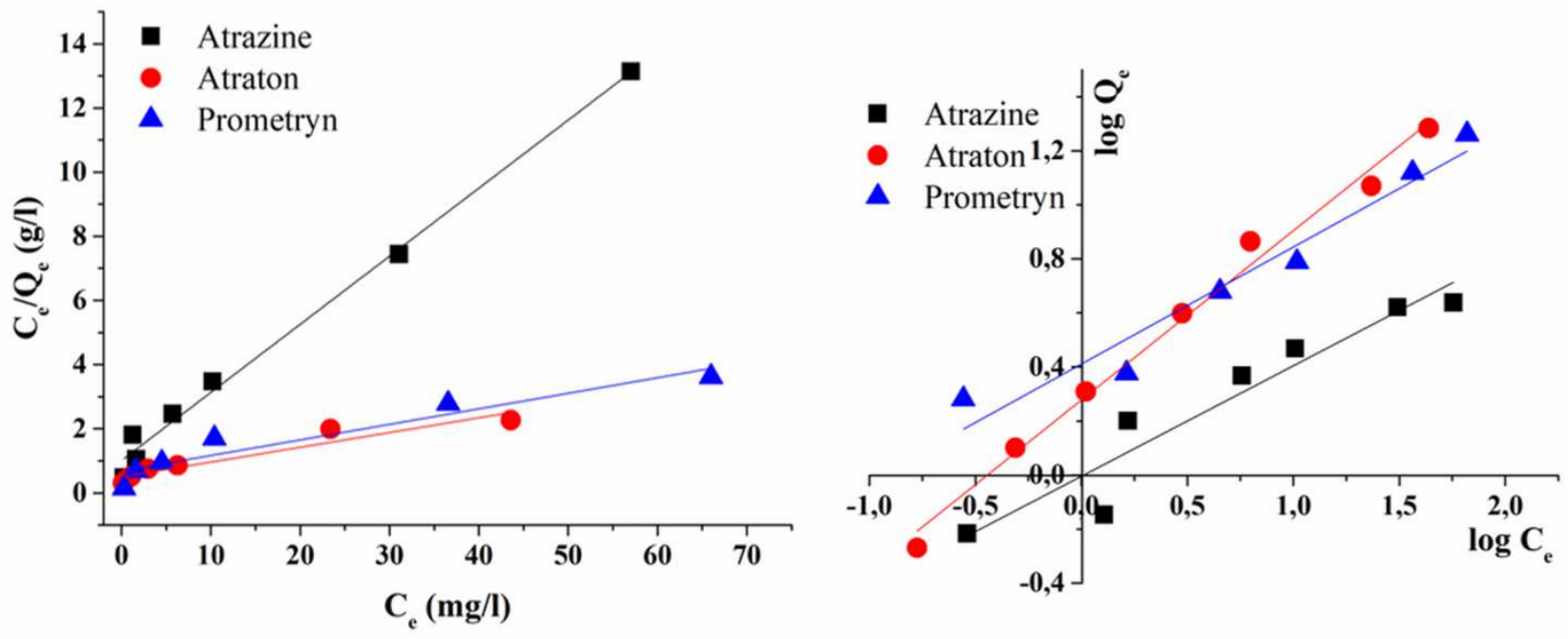
Figure 04 Adsorption isotherm plots described according to the linearized adsorption models of Langmuir (a) and Freundlich (b) and reported for all the involved triazine.

509 The adsorption parameters obtained by applying both models to each of the examined herbicide and the determination coefficients $\left(\mathrm{R}^{2}\right)$ of the linear fits are summarized in Table 03.

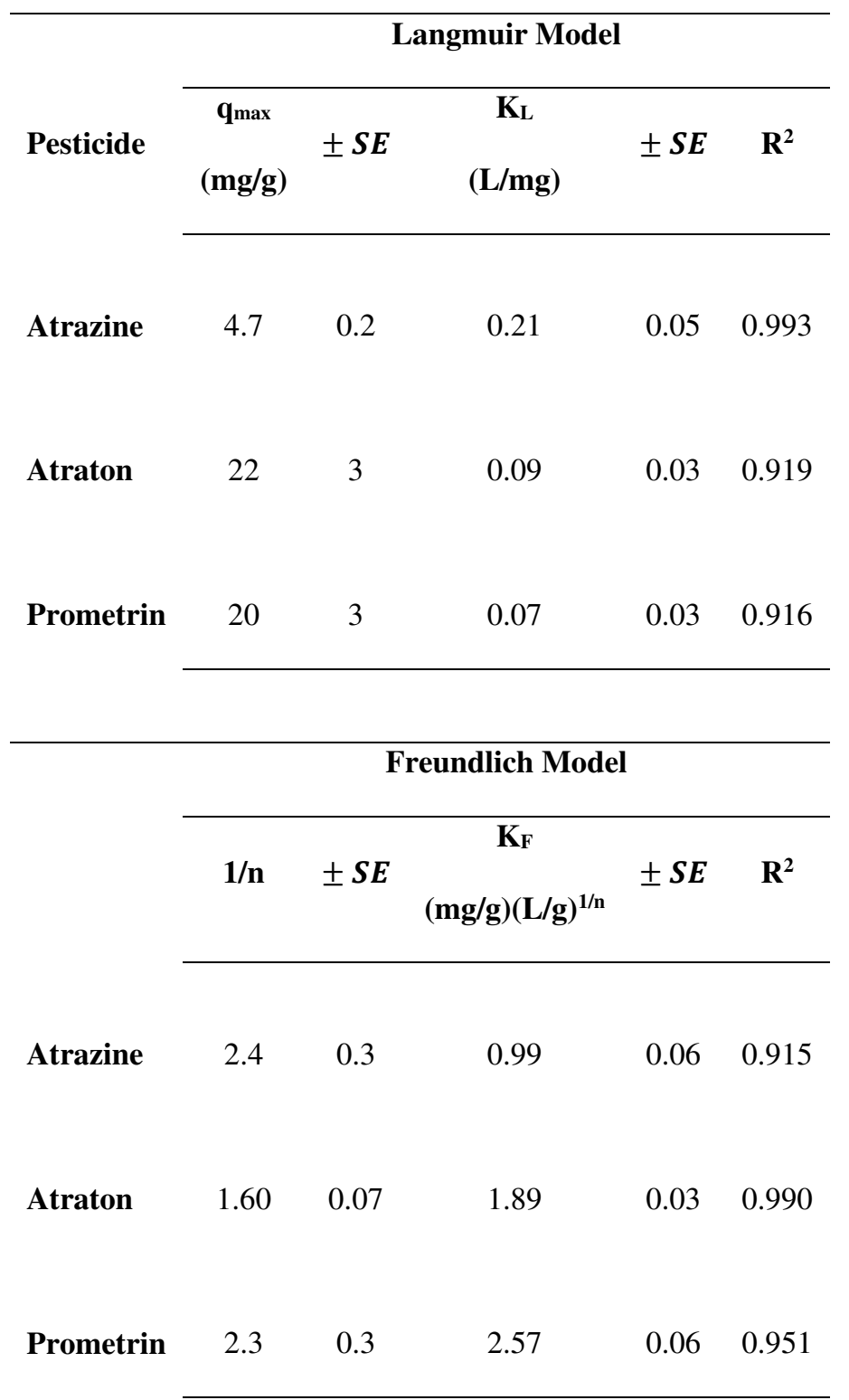

Table 03 Adsorption parameters computed following the linearized Langmuir $\left(\mathrm{q}_{\max } ; \mathrm{K}_{\mathrm{L}}\right)$ and Freundlich $\left(1 / \mathrm{n} ; \mathrm{K}_{\mathrm{F}}\right)$

512 models, their respective Standard Errors (SE) and determination coefficients $\left(\mathrm{R}^{2}\right)$ are reported and computed for each of the investigated triazine.

514 The values show that the Freundlich equation fitted the adsorption data better than the Langmuir one, but the Langmuir model also fits well especially regarding Atrazine, as evidenced by the high $\mathrm{R}^{2}$. In particular, the goodness of fit of the 516 Freundlich model is evidenced by the $\mathrm{R}^{2}$ values varying between 0.915 for adsorption of the atrazine and 0.990 for adsorption of the Atraton. The agreement between the experimental data and those of the model is also confirmed by the 
small uncertainties calculated on the parameters $1 / \mathrm{n}$ and $\mathrm{K}_{\mathrm{F}}$ (Table 03 ). These findings thus effectively demonstrate the 519 heterogeneous enrichment of the triazines on the rGO edges and a multilayer adsorption on the surface of rGO. In the 520 Freundlich models the values of the parameter $\mathrm{n}$ are more than 1, this fact indicates that the adsorption process is 521 favourable. The observed trends are characterised by an increase in the quantity adsorbed as concentration rise. The 522 tendency to reach saturation can be understood as a measure of the maximum adsorbing capacity of the material. 523 Furthermore, the values of $\mathrm{q}_{\max }$ reported in Table 03 show that Atraton is adsorbed more than Prometryn and Atrazine. 524 Atraton, with its methoxy group, may have a greater affinity towards the rGO film, hydrogen bonds could be established 525 with the groups present in the material and interactions $\pi-\pi$. Prometryn, despite being the most apolar pesticide, shows 526 a significant affinity towards the material. The presence of sulphur atoms in Prometryn could promote the formation of 527 hydrogen bonds with the rGO film. Comparing the data reported in Table 03 it can be noted that the constant $\mathrm{K}_{\mathrm{F}}$ assumes 528 the highest value in the case of Prometryn, which is the most apolar among the studied pesticides. The good adsorption 529 capacity can be due to the strong interactions such as hydrogen bonding, electrostatic attraction, and even $\pi-\pi$ interactions.

530 The Freundlich model is the one that best interpolates the experimental data in cases of adsorption on heterogeneous 531 surfaces, consisting of points in which the heat of adsorption is reduced exponentially with the degree of coverage. In the 532 Freundlich model, the relative quantities adsorbed at the maximum concentration $(50 \mathrm{mg} / \mathrm{l}) \mathrm{are} 4.4 \mathrm{mg} / \mathrm{g}$ for Atrazine, $53319.4 \mathrm{mg} / \mathrm{g}$ for Atraton and $18.4 \mathrm{mg} / \mathrm{g}$ for Prometryn which are in good agreement with the $\mathrm{q}_{\max }$ measured in the Langmuir 534 isotherms (respectively 4.7, 22.0 and $20.6 \mathrm{mg} / \mathrm{g}$ ).

535 Usually, the adsorption of analogous compounds follows the trend predicted by the Lundelius rule, which establishes a 536 general criterion in which a compound is less adsorbable the higher its solubility in the solvent. This can be explained by 537 considering that the higher the solubility, the stronger the solute-solvent bond and, therefore, the lower the adsorption 538 capacity. In our case, however, we find an inverse order of adsorption of the triazines, since Atraton is more adsorbed 539 than Prometryn while Atrazine is the least adsorbed. The solubility of atrazine is the lowest among the compounds studied, 540 and is equal to $33 \mathrm{ppm}$ at $27^{\circ} \mathrm{C}$. In our case we attribute this behaviour to the fact that the adsorbent material still contains 541 a high number of oxygenated sites on its skeleton that can form hydrogen bonds with the analytes. But analysing the 542 structure of the three triazines, it is evident that the triazine ring is common to the three analytes, and forms hydrogen 543 bonds with the rGO, as well as interactions with the aromatic residual graphenic skeleton. The substituent group on 544 the triazine ring of Atrazine is a chlorine, which cannot form hydrogen bonds with the adsorbent, while in the case of 545 Atraton and Prometryn both oxygen and sulphur atoms have the potential to form $\mathrm{H}$ bonds. This could explain the reversed 546 behaviour of the three triazine derivatives. 
547 Compared to the adsorption values of triazines in the literature (Zhang et al. 2015; Boruah et al. 2017), our values are 548 slightly lower, but this work is a promising starting point that can help find a valid strategy even with pollutants having 549 different chemical characteristics with respect to the triazine family.

\section{$550 \quad 4$ Conclusions}

551 Graphene and its derivatives have shown excellent performance for environmental applications due to their excellent 552 adsorption capacity. The key surface properties which influence the adsorption on graphene derivatives are surface area, interactions and hydrogen bonding. The reduced graphene oxide adsorption capacity depends on the surface properties of the adsorbent itself, on the presence of sites available to interact with the pollutants $(\mathrm{H}$ bond and interaction). The reduction process used still leave many oxygen-containing functional groups on the rGO, but also a $\pi$ delocalized electron system that results in a good affinity for aromatic pollutants. This was confirmed by Fourier Transform Infrared Spectroscopy (FTIR) and X-ray photoelectron spectroscopy (XPS), where the presence of the characteristic signals of the epoxy and hydroxyl groups is confirmed, despite the slight reduction it has undergone. Those groups, together with the amine pendants present on the triazine rings, may still allow favourable adsorption of the pollutants through hydrogen bonding interactions. Electrostatic interactions between the amino groups of the pesticide and the oxygen containing functionalities of the rGO contributed maximum for adsorption.

A dispersion of the pristine material shows an ultra-high specific surface area but no porosity, but a revolutionary improvement in the adsorption effectiveness of graphenic materials can be achieved by introducing porosity, creating 3D structures by freeze-drying or by forming thick films by evaporation of the solvent. Moreover, the use of thick films, rather than the simple graphene material dispersed in solution, and at the same time the reduction of GO nanosheets allows 567 The coupling of a response surface to an experimental design in which different parameters and different chemicalphysical properties of graphenic materials can be introduced is an original and very versatile approach. From the response surface obtained, the optimal reduction conditions of the material were the reduction at a temperature of $110{ }^{\circ} \mathrm{C}$ for a time

571 It is possible to further optimize the adsorbent material according to the same analytes or to apply the same strategy to study the adsorption of different pollutants. The advantage is in terms of time and experimental tests, since with the strategy just described it is possible to carry out a minimum of preliminary tests to optimize the response surface. This also translates into economic savings, as less adsorbent material is consumed.

575 The Freundlich model fitted best the experimental data. The strength of adsorption of triazines followed the order: Atraton rings aided the adsorption. 
The adsorption yield is not the highest possible, but the graphenic material is a versatile platform, and can also provide for subsequent chemical functionalization, by means of well-known synthetic strategies.

580 This preliminary work can be used to further optimize graphenic materials, choosing which conditions may be the best 581 for the adsorption of different analytes, and preparing the respective GO derivatives that best respond to the adsorption 582 characteristics of the pollutants. By introducing functional groups that modify the surface charge of the material itself we expected an improvement in adsorption capacity of sorbent material.

Acknowledgments We would like to thank all participants who contributed their time and completed laboratory study.

Ethical Approval and Consent to Participate Not applicable

Consent to Publish Not applicable

Authors Contributions GF and FR conceptualized and designed the study and drafted the initial manuscript. GF and PC contributed to the material preparation and characterization. MF, PC and MM conducted adsorption experiments and data analysis. MF, GF and FR contributed to the interpretation of data and critically revised the manuscript. All authors approved the final manuscript as submitted.

Funding This research did not receive any specific grant from funding agencies in the public, commercial, or not-forprofit sectors.

594 Competing Interests The authors declare that they have no competing interests

595 Availability of data and materials The dataset used and/or analysed during the current study are available from the corresponding author on reasonable request.

\section{References}

600 Amini Tapouk F, Nabizadeh R, Nasseri S, et al (2020) Embedding of L-Arginine into graphene oxide (GO) for endotoxin 601 removal from water: Modeling and optimization approach. Colloids Surfaces A Physicochem Eng Asp 607:125491. https://doi.org/10.1016/j.colsurfa.2020.125491 Andrade MB, Santos TRT, Silva MF, et al (2019) Graphene oxide impregnated with iron oxide nanoparticles for the 604 removal of atrazine from the aqueous medium. Sep Sci Technol 54:2653-2670. https://doi.org/10.1080/01496395.2018.1549077

606 Bardestani R, Patience GS, Kaliaguine S (2019) Experimental methods in chemical engineering: specific surface area and 607 pore size distribution measurements-BET, BJH, and DFT. Can J Chem Eng 97:2781-2791. 
Berhane TM, Levy J, Krekeler MPS, Danielson ND (2017) Kinetic sorption of contaminants of emerging concern by a

palygorskite-montmorillonite

filter

medium.

Chemosphere

176:231-242.

611 https://doi.org/https://doi.org/10.1016/j.chemosphere.2017.02.068

612 Bethsass J, Colangelo A (2006) European Union Bans Atrazine, While the United States Negotiates Continued Use. Int 613 J Occup Environ Health 12:260-267. https://doi.org/10.1179/oeh.2006.12.3.260

614 Bonetto LR, Crespo JS, Guégan R, et al (2021) Removal of methylene blue from aqueous solutions using a solid residue 615 of the apple juice industry: Full factorial design, equilibrium, thermodynamics and kinetics aspects. J Mol Struct 616 1224:129296. https://doi.org/https://doi.org/10.1016/j.molstruc.2020.129296

617 Boruah PK, Sharma B, Hussain N, Das MR (2017) Magnetically recoverable Fe3O4/graphene nanocomposite towards 618 efficient removal of triazine pesticides from aqueous solution: Investigation of the adsorption phenomenon and specific 619 ion effect. Chemosphere 168:1058-1067. https://doi.org/10.1016/j.chemosphere.2016.10.103

620 Carolin CF, Kumar PS, Saravanan A, et al (2017) Efficient techniques for the removal of toxic heavy metals from aquatic 621 environment: A review. J Environ Chem Eng 5:2782-2799. https://doi.org/https://doi.org/10.1016/j.jece.2017.05.029

622 Catanesi M, Panella G, Benedetti E, et al (2018) YAP/TAZ mechano-transduction as the underlying mechanism of 623 neuronal differentiation induced by reduced graphene oxide. Nanomedicine (Lond) 13:3091-3106. 624 https://doi.org/10.2217/nnm-2018-0269

625 D'Archivio AA, Maggi MA, Odoardi A, et al (2018) Adsorption of triazine herbicides from aqueous solution by 626 functionalized multiwall carbon nanotubes grown on silicon substrate. Nanotechnology 29:65701. 627 https://doi.org/10.1088/1361-6528/aaa0a0

628 D’Archivio AA, Incani A, Mazzeo P, Ruggieri F (2009) Adsorption of s-triazines onto polybenzimidazole: a quantitative 629 structure-property relationship investigation. Anal Chim Acta 650:175-182. https://doi.org/10.1016/j.aca.2009.07.048

630 de Souza Antonio R, Guerra ACS, de Andrade MB, et al (2020) Application of graphene nanosheet oxide for atrazine 631 adsorption in aqueous solution: synthesis, material characterization, and comprehension of the adsorption mechanism. 632 Env Sci Pollut Res Int. https://doi.org/10.1007/s11356-020-10693-4

633 Frank R, Sirons GJ (1979) Atrazine: Its use in corn production and its loss to stream waters in Southern Ontario, 1975634 1977. Sci Total Environ 12:223-239. https://doi.org/https://doi.org/10.1016/0048-9697(79)90088-3

635 Freundlich HMF (1906) Over the adsorption in solution. J Phys Chem 57:1100-1107

636 Geim AK, Novoselov KS (2007) The rise of graphene. Nat Mater 6:183-191. https://doi.org/10.1038/nmat1849

637 Ghasemi O, Mehrdadi N, Baghdadi M, et al (2020) Spilled oil absorption from Caspian sea water by graphene/chitosan 638 nano composite. Energy Sources Part a-Recovery Util Environ Eff 42:2856-2872. 639 https://doi.org/10.1080/15567036.2019.1618995 
Jablonowski ND, Schäffer A, Burauel P (2011) Still present after all these years: Persistence plus potential toxicity raise 641 questions about the use of atrazine. Environ Sci Pollut Res 18:328-331. https://doi.org/10.1007/s11356-010-0431-y

642 Jeevanantham S, Saravanan A, Hemavathy R V, et al (2019) Removal of toxic pollutants from water environment by 643 phytoremediation: A survey on application and future prospects. Environ Technol Innov 13:264-276. 644 https://doi.org/https://doi.org/10.1016/j.eti.2018.12.007

645 Jung M, Ahn K, Lee Y, et al (2001) Evaluation on the adsorption capabilities of new chemically modified polymeric 646 adsorbents with protoporphyrin IX. J Chromatogr A 917:87-93. https://doi.org/https://doi.org/10.1016/S0021647 9673(01)00673-2

648 Kucka M, Pogrmic-Majkic K, Fa S, et al (2012) Atrazine acts as an endocrine disrupter by inhibiting cAMP-specific 649 phosphodiesterase-4. Toxicol Appl Pharmacol 265:19-26. https://doi.org/https://doi.org/10.1016/j.taap.2012.09.019

650 Langmuir I (1916) The constitution and fundamental properties of solids and liquids. Part I. Solids. J Am Chem Soc 651 38:2221-2295. https://doi.org/10.1021/ja02268a002

652 Lingamdinne LP, Vemula KR, Chang YY, et al (2020) Process optimization and modeling of lead removal using iron 653 oxide nanocomposites generated from bio-waste mass. Chemosphere 243:125257. 654 https://doi.org/https://doi.org/10.1016/j.chemosphere.2019.125257

655 Lu F, Astruc D (2020) Nanocatalysts and other nanomaterials for water remediation from organic pollutants. Coord Chem 656 Rev 408:213180. https://doi.org/https://doi.org/10.1016/j.ccr.2020.213180

657 Lundstedt T, Seifert E, Abramo L, et al (1998) Experimental design and optimization. Chemom Intell Lab Syst 42:3-40. 658 https://doi.org/Doi 10.1016/S0169-7439(98)00065-3

659 MacLennan PA, Delzell E, Sathiakumar N, et al (2002) Cancer Incidence Among Triazine Herbicide Manufacturing 660 Workers. J Occup Environ Med 44:

661 Miller SM, Sweet CW, DePinto J V, Hornbuckle KC (2000) Atrazine and Nutrients in Precipitation: Results from the 662 Lake Michigan Mass Balance Study. Environ Sci Technol 34:55-61. https://doi.org/10.1021/es990486n

663 Mojiri A, Zhou JL, Robinson B, et al (2020) Pesticides in aquatic environments and their removal by adsorption methods. 664 Chemosphere 253:126646. https://doi.org/10.1016/j.chemosphere.2020.126646

665 Nodeh HR, Kamboh MA, Wan Ibrahim WA, et al (2019) Equilibrium, kinetic and thermodynamic study of pesticides 666 removal from water using novel glucamine-calix[4]arene functionalized magnetic graphene oxide. Environ Sci Process 667 Impacts 21:714-726. https://doi.org/10.1039/C8EM00530C

668 Pei Z, Li L, Sun L, et al (2013) Adsorption characteristics of 1,2,4-trichlorobenzene, 2,4,6-trichlorophenol, 2-naphthol 669 and naphthalene on graphene and graphene oxide. Carbon N Y 51:156-163. 670 https://doi.org/https://doi.org/10.1016/j.carbon.2012.08.024 
671 Perez JVD, Nadres ET, Nguyen HN, et al (2017) Response surface methodology as a powerful tool to optimize the 672 synthesis of polymer-based graphene oxide nanocomposites for simultaneous removal of cationic and anionic heavy metal 673 contaminants. Rsc Adv 7:18480-18490. https://doi.org/10.1039/c7ra00750g

674 Perrozzi F, Croce S, Treossi E, et al (2014) Reduction dependent wetting properties of graphene oxide. Carbon N Y 77:. 675 https://doi.org/10.1016/j.carbon.2014.05.052

676 Roberge M, Hakk H, Larsen G (2004) Atrazine is a competitive inhibitor of phosphodiesterase but does not affect the estrogen receptor. Toxicol Lett 154:61-68. https://doi.org/https://doi.org/10.1016/j.toxlet.2004.07.005 Ruggieri F, D’Archivio AA, Foschi M, Maggi MA (2017) Experimental Design in Ion Chromatography: Effect of the Organic Modifier and Complexing Agent on the Retention of Alkaline and Alkaline Earth Ions. Chromatographia 80:853860. https://doi.org/10.1007/s10337-017-3284-4

681 Ruggieri F, D’Archivio AA, Di Camillo D, et al (2015) Development of molecularly imprinted polymeric nanofibers by 682 electrospinning and applications to pesticide adsorption. J Sep Sci 38:1402-1410. 683 https://doi.org/https://doi.org/10.1002/jssc.201500033

684 Ruggieri F, D’Archivio AA, Foschi M, Maggi MA (2020) Multivariate optimization of an analytical method for the 685 analysis of Abruzzo white wines by ICP OES. Anal Methods 12:2772-2778. https://doi.org/10.1039/D0AY00478B 686 Sanderson JT, Boerma J, Lansbergen GWA, van den Berg M (2002) Induction and Inhibition of Aromatase (CYP19) 687 Activity by Various Classes of Pesticides in H295R Human Adrenocortical Carcinoma Cells. Toxicol Appl Pharmacol 688 182:44-54. https://doi.org/https://doi.org/10.1006/taap.2002.9420

689 Singh V, Joung D, Zhai L, et al (2011) Graphene based materials: Past, present and future. Prog Mater Sci 56:1178-1271. 690 https://doi.org/https://doi.org/10.1016/j.pmatsci.2011.03.003

691 Sousa JCG, Ribeiro AR, Barbosa MO, et al (2018) A review on environmental monitoring of water organic pollutants 692 identified by EU guidelines. J Hazard Mater 344:146-162. https://doi.org/https://doi.org/10.1016/j.jhazmat.2017.09.058 693 Tarley CRT, Silveira G, dos Santos WNL, et al (2009) Chemometric tools in electroanalytical chemistry: Methods for 694 optimization based on factorial design and response surface methodology. Microchem J 92:58-67. 695 https://doi.org/https://doi.org/10.1016/j.microc.2009.02.002

696 Thakur K, Kandasubramanian B (2019) Graphene and Graphene Oxide-Based Composites for Removal of Organic 697 Pollutants: A Review. J Chem Eng Data 64:833-867. https://doi.org/10.1021/acs.jced.8b01057

698 Treossi E, Melucci M, Liscio A, et al (2009) High-Contrast Visualization of Graphene Oxide on Dye-Sensitized Glass, 699 Quartz, and Silicon by Fluorescence Quenching. J Am Chem Soc 131:15576-+. https://doi.org/10.1021/ja9055382

700 Vander Heyden Y, Nijhuis A, Smeyers-Verbeke J, et al (2001) Guidance for robustness/ruggedness tests in method 701 validation. J Pharm Biomed Anal 24:723-753. https://doi.org/https://doi.org/10.1016/S0731-7085(00)00529-X 
Vera Candioti L, De Zan MM, Cámara MS, Goicoechea HC (2014) Experimental design and multiple response optimization. Using the desirability function in analytical methods development. Talanta 124:123-138.

704 https://doi.org/https://doi.org/10.1016/j.talanta.2014.01.034

705 Xiao F, Pignatello JJ (2015) Interactions of triazine herbicides with biochar: Steric and electronic effects. Water Res 706 80:179-188. https://doi.org/10.1016/j.watres.2015.04.040

707 Zhang C, Zhang RZ, Ma YQ, et al (2015) Preparation of Cellulose/Graphene Composite and Its Applications for Triazine 708 Pesticides Adsorption from Water. Acs Sustain Chem Eng 3:396-405. https://doi.org/10.1021/sc500738k

709 Zhang S, Wang H, Liu J, Bao C (2020) Measuring the specific surface area of monolayer graphene oxide in water. Mater 710 Lett 261:127098. https://doi.org/https://doi.org/10.1016/j.matlet.2019.127098

711 Zhang WJ, Ruan GH, Li XX, et al (2019) Novel porous carbon composites derived from a graphene-modified high712 internal- phase emulsion for highly efficient separation and enrichment of triazine herbicides. Anal Chim Acta 1071:17713 24. https://doi.org/https://doi.org/10.1016/j.aca.2019.04.041

714 Zhang Y, Cao B, Zhao LL, et al (2018) Biochar-supported reduced graphene oxide composite for adsorption and 715 coadsorption of atrazine and lead ions. Appl Surf Sci 427:147-155. https://doi.org/10.1016/j.apsusc.2017.07.237

716 Zhao GY, Song SJ, Wang C, et al (2011) Determination of triazine herbicides in environmental water samples by high717 performance liquid chromatography using graphene-coated magnetic nanoparticles as adsorbent. Anal Chim Acta 708:155-159. https://doi.org/10.1016/j.aca.2011.10.006

\section{Figure caption}

Figure 01 Triazines used in this work: a) Atrazine, b) Atraton and c) Prometryn; Graphenic materials used as sorbent: d) pristine GO sponge obtained by freeze drying the aqueous dispersion and e) rGO film obtained by simple evaporation of the solvent in a petri dish.

Figure 02 Surface plot a) and related contour plot b) of the percentage of adsorbed atrazine as a function of the reduction process parameters (temperature and time).

Figure 03 a-c) SEM images of rGO film reduced at $110{ }^{\circ} \mathrm{C}$; d) XRD patterns of GO (black line) and rGO film reduced at $110^{\circ} \mathrm{C}$ (red line); e) FTIR spectra of GO and reduced GO at $110^{\circ} \mathrm{C}$; XPS survey of rGO at $110{ }^{\circ} \mathrm{C}(\mathbf{f}$ ) and $\mathrm{C} 1 \mathrm{~s}$ region 734 (g); BET isotherm (h) for GO (black square) and rGO (red circle) and (i) BJH pores average volume and diameter for GO 
737 Figure 04 Adsorption isotherm plots described according to the linearized adsorption models of Langmuir (a) and 738 Freundlich (b) and reported for all the involved triazine.

739 


\section{Figures}

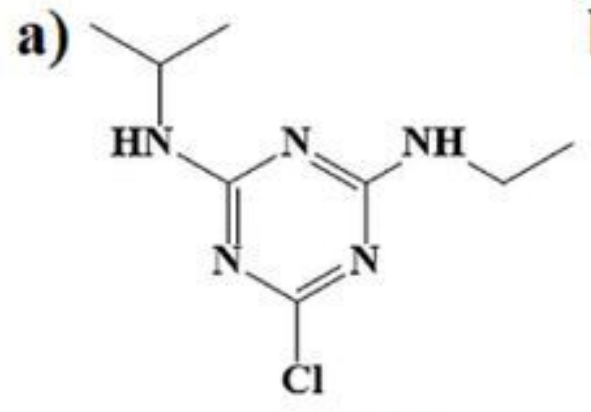

Atrazine

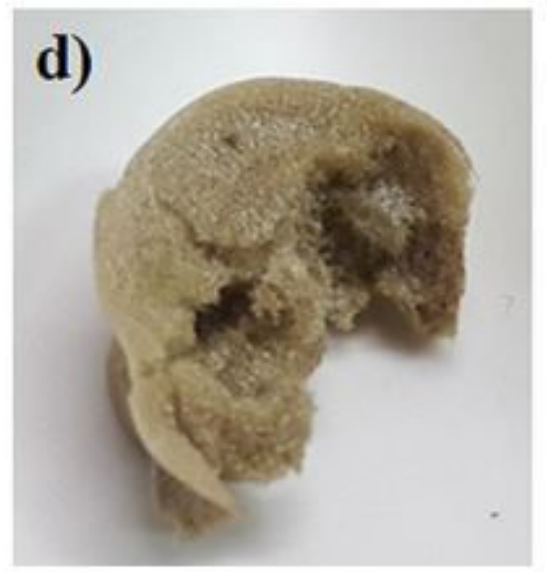

b)

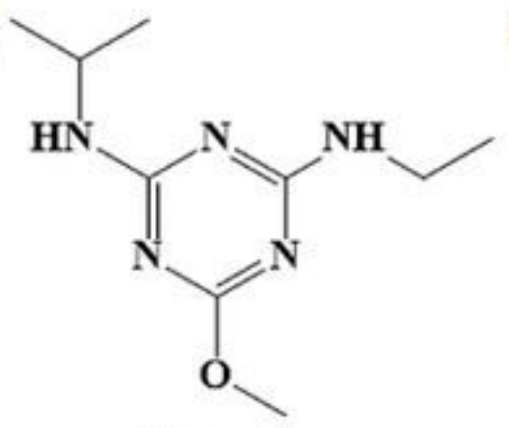

Atraton

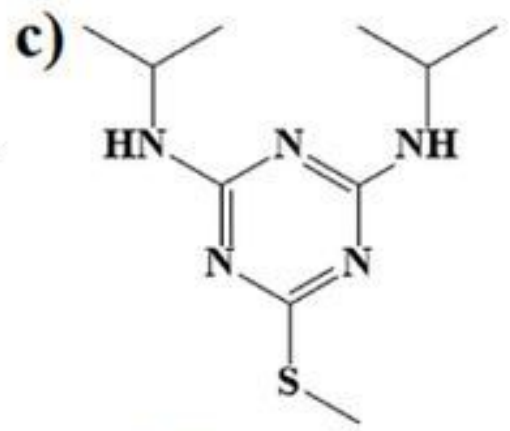

Prometryn

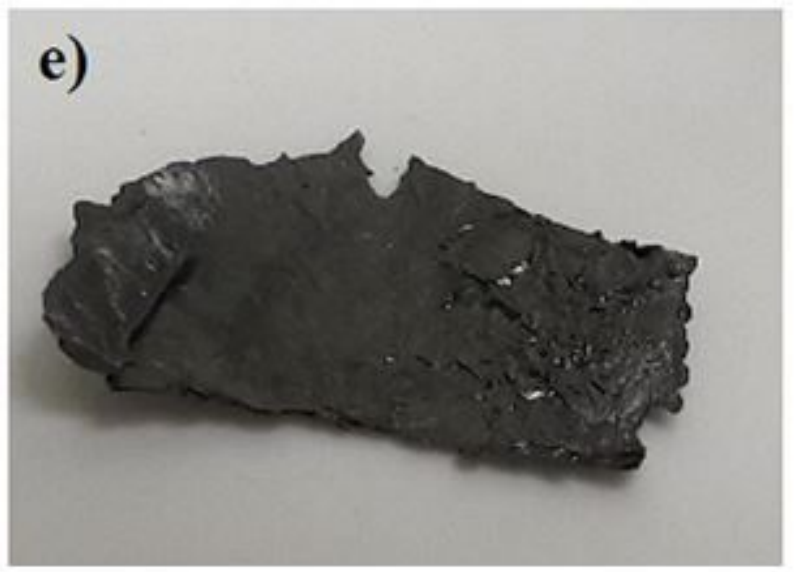

\section{Figure 1}

Triazines used in this work: a) Atrazine, b) Atraton and c) Prometryn; Graphenic materials used as sorbent: d) pristine GO sponge obtained by freeze drying the aqueous dispersion and e) rGO film obtained by simple evaporation of the solvent in a petri dish. 

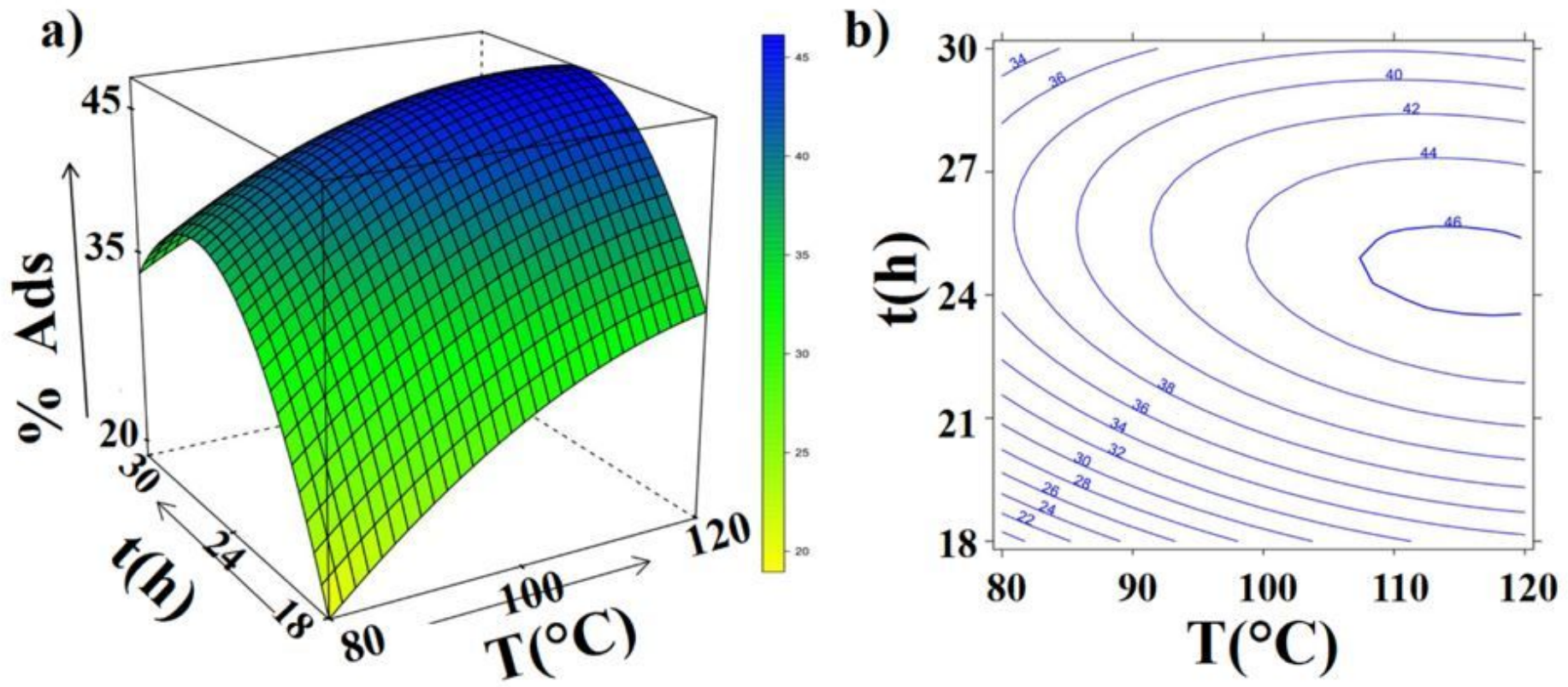

Figure 2

Surface plot a) and related contour plot b) of the percentage of adsorbed atrazine as a function of the reduction process parameters (temperature and time). 

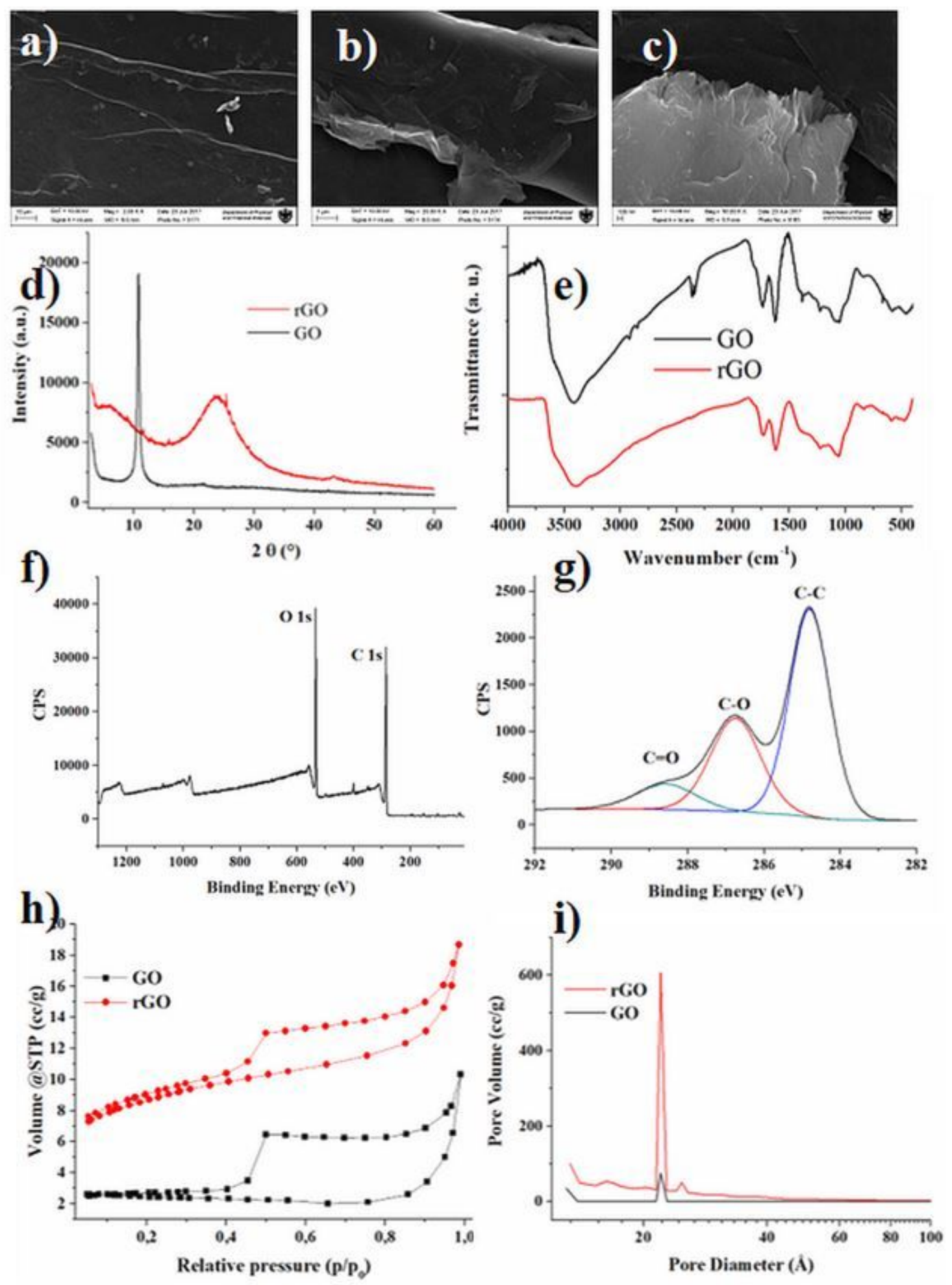

\section{Figure 3}

a-c) SEM images of rGO film reduced at $110^{\circ} \mathrm{C}$; d) XRD patterns of GO (black line) and rGO film reduced at $110{ }^{\circ} \mathrm{C}$ (red line); e) FTIR spectra of GO and reduced GO at $110{ }^{\circ} \mathrm{C}$; XPS survey of $\mathrm{rGO}$ at $110^{\circ} \mathrm{C}(\mathrm{f})$ and $\mathrm{C} 1 \mathrm{~s}$ region (g); BET isotherm (h) for GO (black square) and rGO (red circle) and (i) BJH pores average volume and diameter for GO (black line) and rGO (red line) 

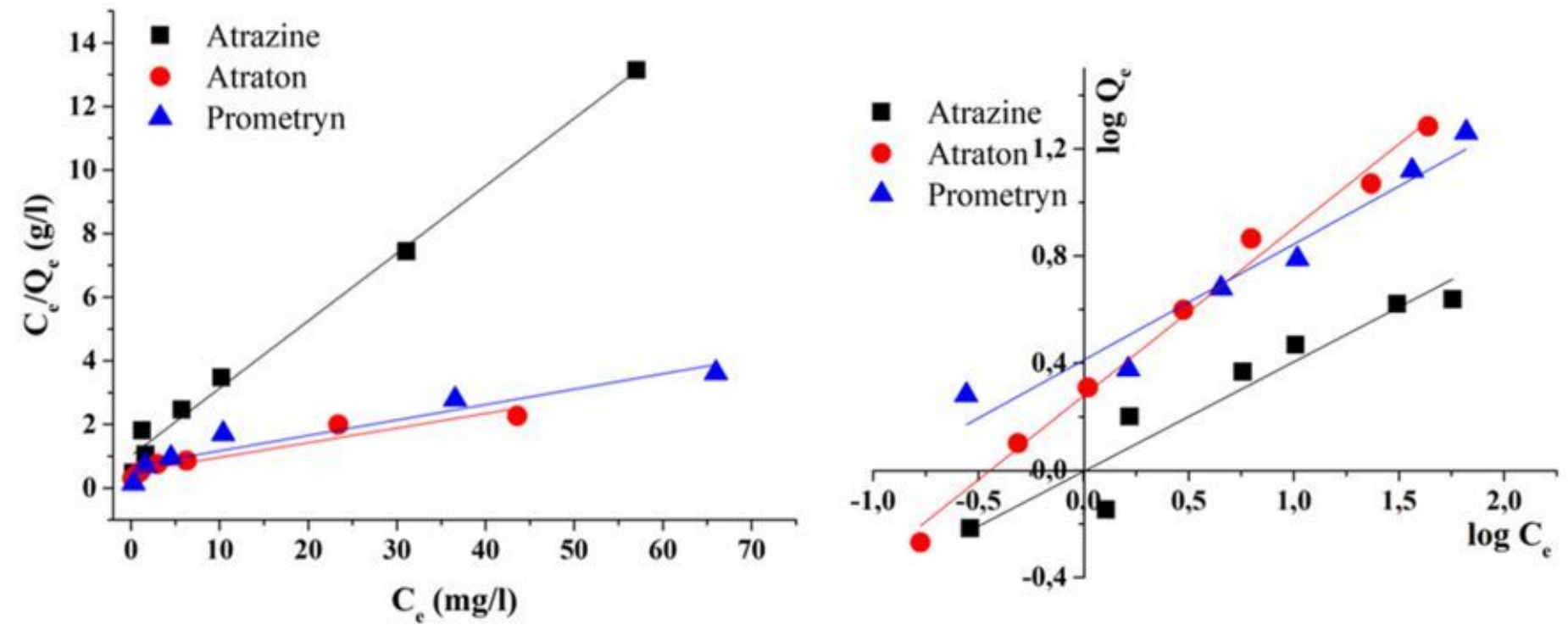

Figure 4

Adsorption isotherm plots described according to the linearized adsorption models of Langmuir (a) and Freundlich (b) and reported for all the involved triazine.

\section{Supplementary Files}

This is a list of supplementary files associated with this preprint. Click to download.

- Abstractlmage.jpg

- SupportingInformationFile.docx 